\title{
Identification of a Person using Multimodal Biometric System
}

\author{
T.C. Sabareeswari \\ M.E-Applied Electronics \\ Anna University, Tirunelveli.
}

\author{
S. Lenty Stuwart \\ Assistant Professor, Department of ECE \\ Anna University, Tirunelveli
}

\begin{abstract}
A biometric identification system is an automatic pattern recognition system that recognizes a person by determining the authenticity of a specific physiological and/or behavioral characteristic (biometric) possessed by that person. Unimodal biometric systems often face significant limitations due to sensitivity to noise, intraclass variability, data quality, nonuniversality, and other factors. To improve the performance of individual matchers in such situations may not prove to be highly effective. Multibiometric systems seek to alleviate some of these problems by providing multiple pieces of evidence of the same identity. These systems help achieve an increase in performance that may not be possible using a single-biometric indicator. An effective fusion scheme that combines information presented by multiple domain experts based on the rank-level fusion integration method. The developed multimodal biometric system possesses a number of unique qualities, starting from utilizing principal component analysis and Fisher's linear discriminant methods for individual matchers (face, ear, and signature) identity authentication and utilizing the novel rank-level fusion method in order to consolidate the results obtained from different bio- metric matchers. The ranks of individual matchers are combined using the highest rank, Borda count, and logistic regression approaches. The results indicate that fusion of individual modalities can improve the overall performance of the biometric system, even in the presence of low quality data. Moreover better performance can be obtained by using ICP algorithm for ear database.
\end{abstract}

Keywords - Biometric identification system, logistic regression, multibiometric system, pattern recognition, principal component analysis (PCA), rank-level fusion.

\section{INTRODUCTION}

Biometric information system is one of the finest examples of computer system that tries to imitate the decisions that humans make in their everyday life, specifically concerning people identification and matching tasks. In this quest, the biometric systems evolved from simple single-feature-based models to a complex decision-making mechanism that utilize artificial intelligence, neural networks, complex decision making schemes, and multiple biometric parameters extracted and combined in an intelligent way. The main goal and contribution of this system is to present a comprehensive analysis of various biometric fusion techniques in combination with advanced biometric feature extraction mechanisms that improve the performance of the biometric information system in the challenging and not resolved problem of people identification. Physiological biometric identifiers include fingerprints, hand geometry, ear patterns, eye patterns (iris and retina), facial features, and other physical characteristics. Behavioral identifiers include voice, signature, typing patterns, and others. In recent years, biometric authentication has seen considerable improvements in reliability and accuracy, with some biometrics offering reasonably good overall performance. Multibiometric systems can significantly improve the recognition performance in addition to improving population coverage, deterring spoof attacks, increasing the degrees of freedom, and reducing the failure-to-enroll rate.

The key to successful multibiometric system is in an effective fusion scheme, which is necessary to combine the information presented by multiple domain experts. The goal of fusion is to determine the best set of experts in a given problem domain and devise an appropriate function that can optimally combine the decisions rendered by the individual experts. A decision made by a biometric system is either a "genuine individual" type of decision or an "impostor" type of decision. [1] The genuine distribution and the impostor distribution, which are used to establish the following two error rates.

1) False acceptance rate (FAR), which is defined as the probability of an impostor being accepted as a genuine individual. It is measured as the fraction of impostor score exceeding the predefined threshold.

2) False rejection rate (FRR), which is defined as the probability of a genuine individual being rejected as an impostor. It is measured as the fraction of genuine score below the predefined threshold.

A FAR of zero means that no impostor is accepted as a genuine individual. Sometimes, another term, genuine accept rate (GAR), is used to measure the accuracy of a biometric system.

The rest of the paper is organised as follows: Related work for recognising in section 2 , proposed work in section 3 , results and discussion in section 4 and conclusion in section 5 .

\section{RELATED WORK}

In 1998, a bimodal approach was proposed by Hong and Jain for a PCA based face and a

minutiae-based fingerprint identification system with a fusion method at the decision level. [1] At a FAR of $0.01 \%$, the monomodal systems obtained FRRs of $61.2 \%$ and $10.6 \%$ for face and fingerprint, respectively. For the same FAR, the fusion approach obtained an FRR of $6.6 \%$.

In 2000, Frischholz and Dieckmann [2] developed a commercial multimodal approach, BioID, for a model-based face classifier, a VQ-based voice classifier, and an opticalow based lip movement classifier for verifying persons. Lip motion and face images were extracted from a video sequence and the voice from an audio signal. Accordingly to the security level, experiments on 150 persons demonstrated a decrease below $1 \%$ of the FAR

In 2003, Fierrez-Aguilar and Ortega-Garcia [3] proposed a multimodal approach including a face verification 
system based on a global appearance representation scheme, a minutiae-based fingerprint verification system, and an online signature verification system based on HMM modeling of temporal functions with fusion methods, i.e., sum-rule and support vector machine (SVM) user independent and user dependent, at the score level. The EERs of the face, the online signature, and the finger print verification systems were $10 \%, 4 \%$, and $3 \%$, respectively, while the sum-rule, the SVM user-independent, and the SVM user-dependent fusion approaches obtained EERs of $0.5 \%$, $0.3 \%$, and $0.05 \%$, respectively.

In the same year, Kumar et al [4] proposed a multimodal approach for palmprint and hand geometry, with fusion methods at the feature level by combining the feature vectors by concatenation, and the matching score level by using max rule. Only the fusion approach at the matching score level outperforms the monomodal systems. For an FRR of $1.41 \%$, the multimodal approach obtained a FAR of $0 \%$, while the palmprint-based verification system, the best monomodal approach in this study, obtained a FAR of $4.49 \%$ at an FRR of $2.04 \%$.

In 2004, Toh et al. [6] developed a system using hand geometry, fingerprint, and voice biometric with weightedsum rule-based match-score-level fusion. They treated the multimodal biometric decision fusion problem as a two-stage problem: learning and decision. Experiments on fingerprint, speech, and hand-geometry biometric data showed that local learning alone can improve verification ERRs of about $50 \%$.

In 2005, Snelick et al. [7] developed a multimodal approach for face and fingerprint, with fusion methods at the score level. Three fingerprint recognition commercial systems and one face recognition commercial system were used in this study. The EERs of the best fingerprint system and the face recognition system were $2.16 \%$ and $3.76 \%$, respec tively, while the max-score fusion approach on quadric-line quadric normalized scores obtained an EER of $0.63 \%$.

\section{PROPOSED WORK}

This section deals with the development procedures of the proposed multimodal biometric system through the ranklevel fusion method. Rank-level fusion is a relatively new fusion approach.

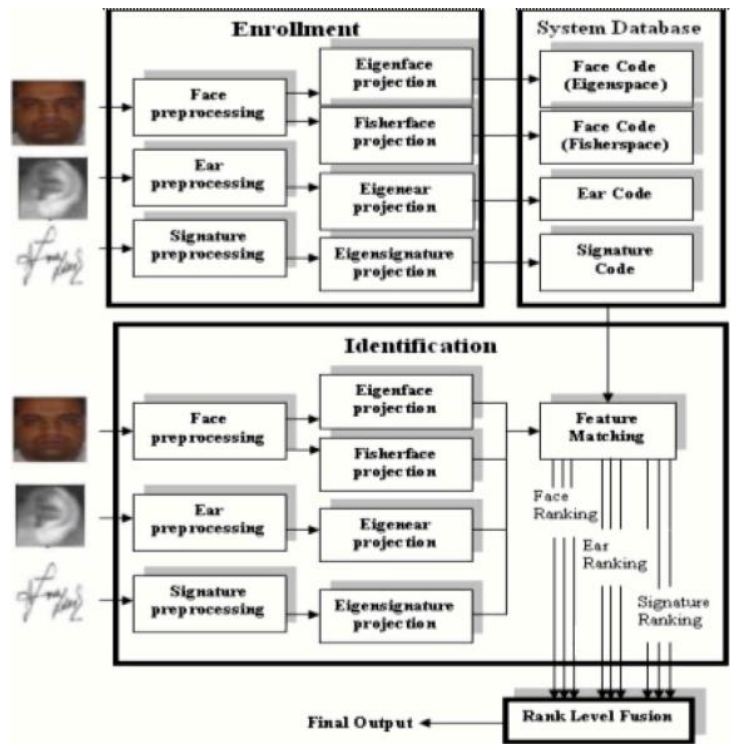

Fig1 : Block diagram of Multibiometric system.
The goal of rank-level fusion is to consolidate the rank output by individual biometric subsystems (matchers) in order to derive a consensus rank for each identity. There are basically two types of recognition approaches appearance based and model based. PCA and LDA are examples of appearance-based recognition approaches. PCA is a statistical method which involves analysis of n- -dimensional data. PCA observes correspondence between different dimensions and determines principal dimensions, along which the variation of the data is high.

\subsection{Recognition Using Eigenimage}

Eigenimage feature extraction is based on the $\mathrm{K}-\mathrm{L}$ transform [8] and is used to obtain the most important features from the face, ear, and signature subimages in our system. These features are obtained by projecting the original subimages into the corresponding subspaces. The process of obtaining these subspaces and projecting the subimages into them is identical for all subspaces. The system is first initialized with a set of training images. Eigenvectors and eigenvalues are computed on the covariance matrix of these images according to the standard procedure. The higher the eigenvalue, the more characteristic features of an image the particular eigenvector describes. Eigenimages with low eigenvalues can be omitted. Finally, the known images are projected onto the image space, and their weights are stored. This process is repeated as necessary. The steps for the recognition process

1) Project the test image into the eigenspace, and measure the distance between the unknown image's position in the eigenspace and all the known image's positions in the eigenspace.

2) Select the image closest to the unknown image in the eigenspace as the match.

\subsection{Recognition Using Fisherface}

Eigenspace representation is very sensitive to image conditions such as background noise, image shift, occlusion of objects, scaling of the image, and illumination change. When substantial changes in illumination and expression are present in any image, much of the variation in data is due to these changes [9], and the eigenimage technique, in this case, cannot give highly reliable results.

To overcome this a new method called fisherface method is adopted. The fisherface method uses both PCA and LDA to produce a subspace projection matrix, similar to that used in the eigen face method. However, the fisherface method is able to take advantage of within-class information, minimizing variation within each class, yet still maximizing class separation. Scatter matrices, representing the withinclass $(\mathrm{S} \mathrm{W})$, between-class $(\mathrm{S} \quad \mathrm{B})$, and total $(\mathrm{S} \quad \mathrm{T})$ distributions are

$$
\begin{aligned}
& \mathrm{S}_{\mathrm{W}}=\sum_{i=1}^{C} \sum_{\Gamma_{k} \varepsilon X_{i}}\left(\Gamma k-\Psi_{i}\right)\left(\Gamma k-\Psi_{i}\right)^{T} \\
& \mathrm{~S}_{B}=\sum_{i=1}^{C}\left|X_{i}\right|\left(\Psi_{i}-\Psi\right)\left(\Psi_{i}-\Psi\right)^{T}
\end{aligned}
$$




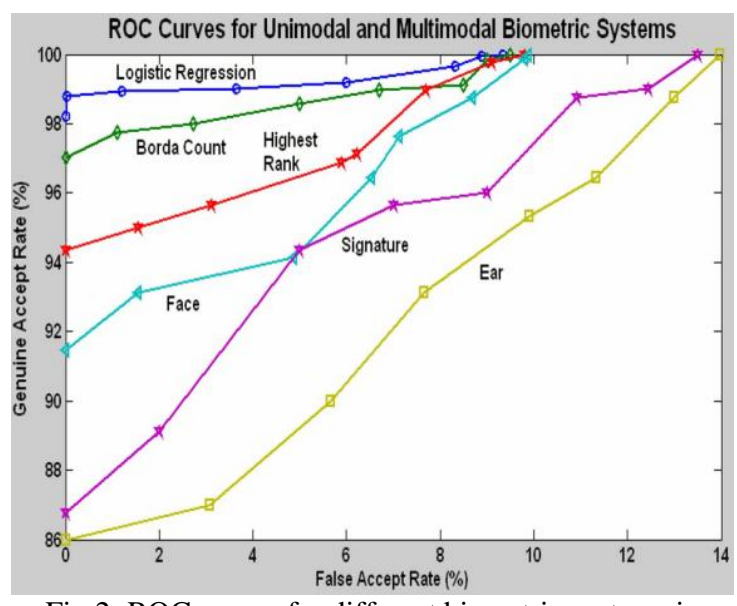

Fig 2: ROC curves for different biometric systems in terms of GAR and FAR

$\mathrm{S}_{T}=\sum_{n=1}^{M}\left(\Gamma_{n}-\Psi\right)\left(\Gamma_{n}-\Psi\right)^{T}$

Where, $\quad \Psi=(1 / M) \sum_{n=2}^{M} \Gamma_{n}$ is the average image vector of the entire training set and $\Psi_{i}=\left(1 /\left|X_{i}\right|\right) \sum_{\Gamma_{i} \in X_{i}} \Gamma_{i}$ is the average of each individual class $\mathrm{X}_{i}$.

\subsection{Fusing Rank Information}

This can be applied after those methods. After getting the identification results with ranks by the FLD based unimodal system, its compared with the results obtained from the eigenface-based subsystem. The ranked output of these three matchers is then consolidated by using the highest rank, Borda count, and logistic regression methods. Choose 0.3, 0.4 , and 0.3 as the weights for face, ear, and signature, respectively. The more the weight, the less the recognition rate of the system. This means that the ear matcher gives us less accurate results than the face or signature matchers. These weights are chosen by reviewing the previous research, examining the quality of the database and by consequently executing the system.

\section{RESULTS AND DISCUSSION}

This section consists of a description of the databases used for verifying the developed system performance and the description of the extensive experimental setup. Multibiometric system is implemented in MATLAB 7.0 on a Pentium-IV Windows XP workstation. The system is graphical-user-interface based and menu driven. The necessary image preprocessing can be easily done by selecting the image directory. Also, the threshold for recognition of face, ear, and signature and the weights for the logistic regression method (for fusion) can be changed in run time by selecting proper menus. The top-ranked matched images will be shown in the case of single-biometric recognition, as well as multiple biometric recognition after fusion. For convenient use of the system, the proper database, consisting of different subdirectories of training faces, ears, and signatures, will be automatically connected to the system after execution.
The multiple biometrics of a single person for the final result can also be chosen by only selecting the directory containing the face, ear, and signature images of that person. To make the system robust, thresholds are chosen in such a way that the system can differentiate between a face and a nonface image.

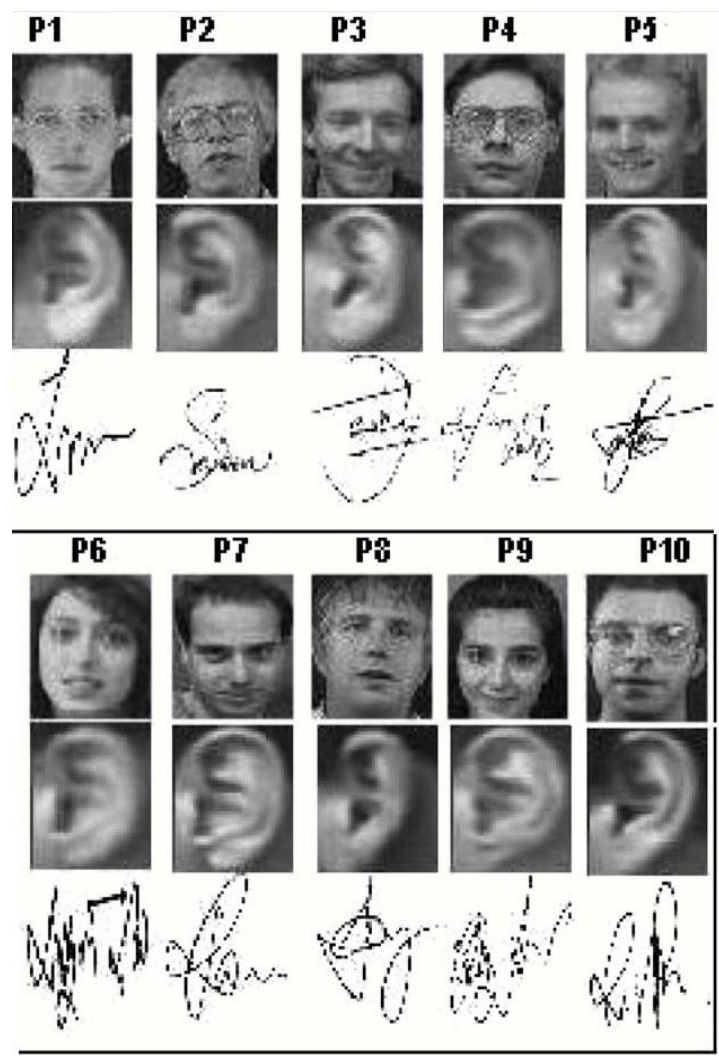

Fig 3: Sample of the virtual multimodal database

Training database plays a very important role in achieving better recognition performance from a biometric system. In a multibiometric system, it is quite often that the database used is not the true database. i.e., different biometric traits are collected from the same person; instead, the said database is a virtual database which contains records which are created by consistently pairing a user from one unimodal database (e.g., face) with a user from another database (e.g., iris).

In this section various eigenimage techniques and the fisher face technique are compared in terms of FAR and GAR. 


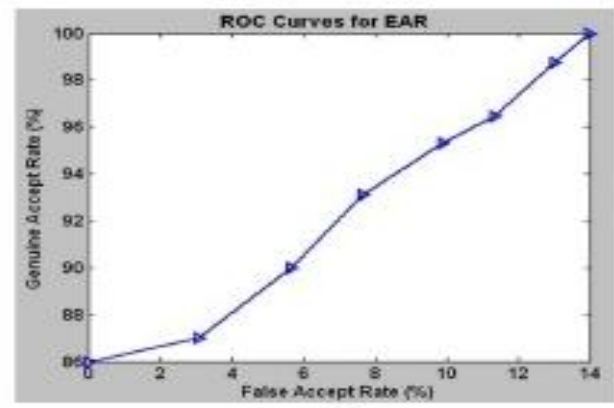

(a)

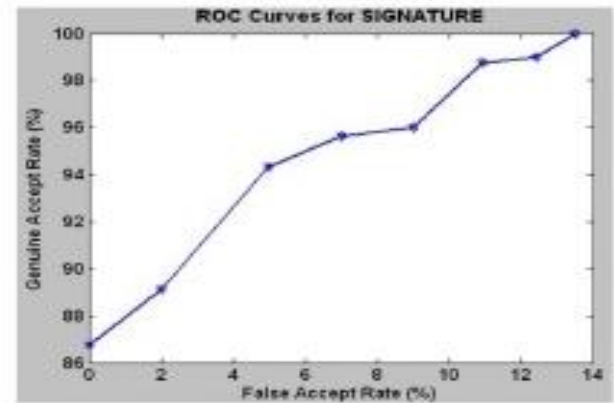

(b)

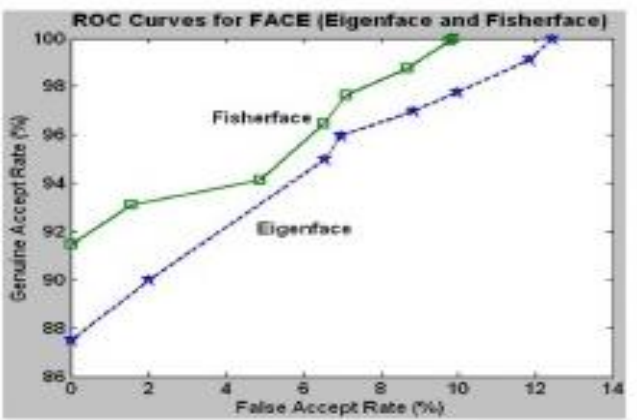

(c)

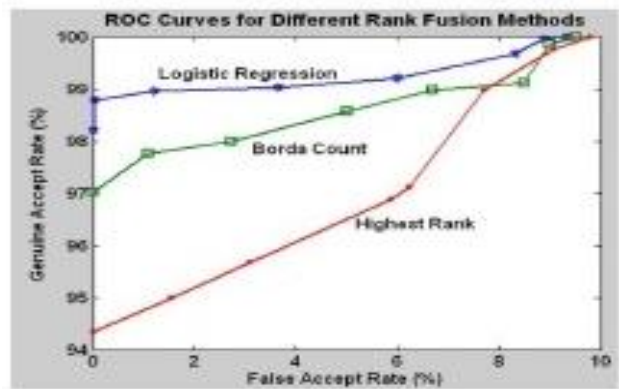

(d)

Fig 4: ROC curves for different biometric systems - for (a) ear, (b) signature (c) face, and (d) three different approaches for rank level fusion methods

From the results shown in the graph of Fig.3, it is clear that fisherface works more efficiently than eigenface. Among the three eigenimage methods, face-based recognition provides the best performance. Between eigenear and eigensignature methods, the eigensignature method is slightly better than the eigenear method. Variations in lighting conditions, facial expression, and even small change in orientation can cause the face image of a person to change from one form to another. In face database there are ten face images of the same person but with expression, illumination, and orientation changes. The fisherface method takes care of these changes, while the eigenimage method does not. Therefore, in this system, a better recognition performance is obtained by the fisherface method.

Fig. 4 shows the combined receiver operating characteristic (ROC) curves under one graph. From this figure, it is clear that the error rate would be reasonably high without incorporating any fusion method. Significant performance gain can be achieved with the combination of rank information of different monomodal experts. The best performance that we have received from this system is using the logistic regression approach of the rank-level fusion method. In this method, assigning different weights to individual matchers based on their accuracy plays a significant role in determining the final result. The second best result is obtained through the Borda count method. This method is similar to the logistic regression method, except that there is no weight-assigning procedure in this method. This leads to a vital issue on the performance of the biometric system.

\section{CONCLUSION}

Multibiometrics is a new and exciting area of information science research for accurate and reliable personal information representation for matching. This is specifically focused to find a good combination of multiple biometric traits and various fusion methods to get the optimal identification results.

In this a comparison between various rank-level fusion methods are obtained. Between the three rank-level fusion approaches, the logistic regression method gives us the better performance in terms of error rates. The main reason for this is that, in this approach, weights are assigned to different matchers according to their performance.

In future, a better result can be obtained by improving the genuine acceptance rate and decreasing the false acceptance rate. This can be done by using some specific algorithm for each database.

\section{REFERENCES}

[1] L. Hong and A. K. Jain, "Integrating faces and fingerprints for personal identification," IEEE Trans. Pattern Anal. Mach. Intell. , vol. 20, no. 12, pp. 12951307, Dec. 1998

[2] R. Frischholz and U. Dieckmann, "BiolD: A multimodal biometric identification system," Computer,vol.33,no.2, pp.64-68,Feb,2000

[3] J. Fierrez-Aguilar, J. Ortega-Garcia, D. Garcia-Romero, and J. Gonzalez Rodriguez, "A comparative evaluation of fusion strategies for multimodal biometric verification,"in Proc. $4^{\text {th }}$ Int, Conf,Audio-video-based Biometric Person Authentication, J. Kittler and M. Nixon, Eds., 2003 vol. LNCS 2688, pp. 830-837

[4] A. Kumar, D. C. M. Wong, H. C.Shen1, and A. K. Jain, "Personal verification using palmprint and hand geometry biometric,"in Proc. 4th Int. Conf. Audio- Video-Based Biometric Person Authentication, J. Kittler and M. Nixon, Eds., 2003 vol. LNCS 2688, pp 668-678

[5] T. Wang, T. Tan, and A. K. Jain, "Combining face and iris biometrics for identity verification," in Proc. 4th Int. Conf. Audio- Video-Based Biometric Person Authentication, J. Kittler and M. Nixon, Eds., 2003 vol. LNCS 2688, pp. 805-813 
[6] K. A. Toh, X. D. Jiang, and W. Y. Yau, "Exploiting global and local decisions for multi-modal biometrics verification," IEEE Trans. Signal Process. , vol. 52, no. 10, pp. 3059-3072, Oct. 2004

[7] R. Snelick, U. Uludag, A. Mink, M. Indovina, and A. K. Jain, "Large scale evaluation of multimodal biometric authentication using state-of the-art systems," IEEE Trans. Pattern Anal. Mach. Intell , vol. 27, no. 3, pp. 450455, Mar. 2005
[8] M. Turk and A. Pentland, "Eigenfaces for recognition," J. Cogn Neurosci. , vol. 3, no. 1, pp. 71-86, 1991

[9] T. Heseltine, N. Pears, J. Austin, and Z. Chen, "Face recognition: A comparison Comput.: Tech. Appl. of appearance-based approaches," in Proc. 7th Digit. Image, C. Sun, H. Talbot, S. Ourselin, and T. Adriaansen Eds., Sydey, Australia, 2003, pp. 59-68. 